\title{
Diffusion in Porous Media: Phenomena and Mechanisms
}

\author{
Daniel M. Tartakovsky ${ }^{1}$ (D) Marco Dentz ${ }^{2}$
}

Received: 10 July 2018 / Accepted: 6 March 2019 / Published online: 18 March 2019

(c) Springer Nature B.V. 2019

\begin{abstract}
Two distinct but interconnected approaches can be used to model diffusion in fluids; the first focuses on dynamics of an individual particle, while the second deals with collective (effective) motion of (infinitely many) particles. We review both modeling strategies, starting with Langevin's approach to a mechanistic description of the Brownian motion in free fluid of a point-size inert particle and establishing its relation to Fick's diffusion equation. Next, we discuss its generalizations which account for a finite number of finite-size particles, particle's electric charge, and chemical interactions between diffusing particles. That is followed by introduction of models of molecular diffusion in the presence of geometric constraints (e.g., the Knudsen and Fick-Jacobs diffusion); when these constraints are imposed by the solid matrix of a porous medium, the resulting equations provide a pore-scale representation of diffusion. Next, we discuss phenomenological Darcy-scale descriptors of pore-scale diffusion and provide a few examples of other processes whose Darcy-scale models take the form of linear or nonlinear diffusion equations. Our review is concluded with a discussion of fieldscale models of non-Fickian diffusion.
\end{abstract}

Keywords Multiscale $\cdot$ Brownian motion · Langevin equation · Non-Fickian

Mathematics Subject Classification $60 \mathrm{~J} 60 \cdot 60 \mathrm{~J} 70 \cdot 76 \mathrm{~S} 05$

\section{Introduction}

Diffusion is one of the most omnipresent natural phenomena. Its ubiquity is matched by that of porous media; given a sufficient spatial resolution, virtually every solid or soft material (condensed matter) is porous. It therefore comes as no surprise that the earliest reported study of diffusion dealt with diffusion in a porous medium: selective movement of liquids across an

$\triangle$ Daniel M. Tartakovsky

tartakovsky@stanford.edu

Marco Dentz

marco.dentz@csic.es

1 Department of Energy Resources Engineering, Stanford University, 367 Panama Street, Stanford, CA 94305, USA

2 Institute of Environmental Assessment and Water Research, Spanish National Research Council (CSIC), 08034 Barcelona, Spain 
animal bladder observed by Jean Antoine Nollet in 1752 (Narasimhan 1999, Sect. 5.2). Much of the early theoretical understanding of diffusive processes has been derived from studies of solutes in both free (bulk) fluids and fluids in confining geometries, e.g., capillary tubes.

The theory of diffusion follows two distinct but interconnected paths, that of collective (effective) motion of (infinitely many) particles and that of an individual particle. The former is based on Fick's law of diffusion, which was formulated in 1855 by Adolf Fick in direct analogy with Fourier's law of heat conduction in solids: "concentration is analogous to temperature, heat flux is analogous to solute flux and thermal diffusivity is analogous to chemical diffusivity" (Narasimhan 1999). In a companion paper published the same year, Fick studied solute diffusion in a semipermeable membrane by conceptualizing the latter as a collection of one-dimensional capillary tubes (Narasimhan 1999).

Irregular motion of minute charcoal particles was observed in 1784 by Jan Ingenhousz, who reported his observations in a short note on the use of a microscope (van der Pas 1971). Such an irregular motion of inanimate corpuscules was observed, 43 years later, by Robert Brown for the motion of pollen grains (van der Pas 1971). Both authors pointed out that inanimate corpuscules exhibit an irregular, continuous motion as if they were alive. This motion was later termed Brownian motion (van der Pas 1971). The origins of this motion were not understood until 1905-1906 when the studies of Albert Einstein (1905), Marian von Smoluchowski (1906), and William Sutherland (1905) related this irregular motion to the impact of molecular forces exerted by the liquid. A couple years later, Paul Langevin (1908) formalized their work by expressing a particle's motion in terms of Newton's second law and introducing a random force to represent the action of the fluid molecules surrounding the suspended particle.

We provide a brief overview of these two approaches to modeling diffusion in fluids. Section 2 contains a description of Langevin's approach to a mechanistic description of the Brownian motion in free fluid of individual point-size inert particles and its relation to Fick's diffusion equation (Sect. 2.1), as well as its generalizations that account for a finite number of finite-size particles (Sect. 2.2), particle's electric charge (Sect. 2.3), and chemical interactions between diffusing particles (Sect. 2.4). Models of molecular diffusion in the presence of geometric constraints (e.g., the Knudsen and Fick-Jacobs diffusion) are discussed in Sect. 3; when these constraints are imposed by the solid matrix of a porous medium, the resulting equations provide a pore-scale representation of (advection-)diffusion transport. Section 3 also includes a brief introduction to phenomenological Darcy-scale descriptors of these processes; a comparative analysis of systematic upscaling techniques for deriving Darcy-scale models from their pore-scale counterparts can be found elsewhere in this special issue. Section 4 provides examples of other phenomena whose Darcy-scale models employ diffusion-like equations, including single- and multiphase flow and hydrodynamic dispersion. We conclude our review by discussing Darcy-scale models of non-Fickian diffusion in Sect. 5.

\section{Molecular Diffusion in Free Fluid}

\subsection{Fundamentals of Molecular Diffusion}

We consider the motion of inert particles in a host medium, which may be, e.g., a fluid or the surface between a liquid and a gas. We follow in the next section Langevin's explanation of Brownian motion and then show the equivalence to the Fick's diffusion equation following Einstein and Smoluchowski. 


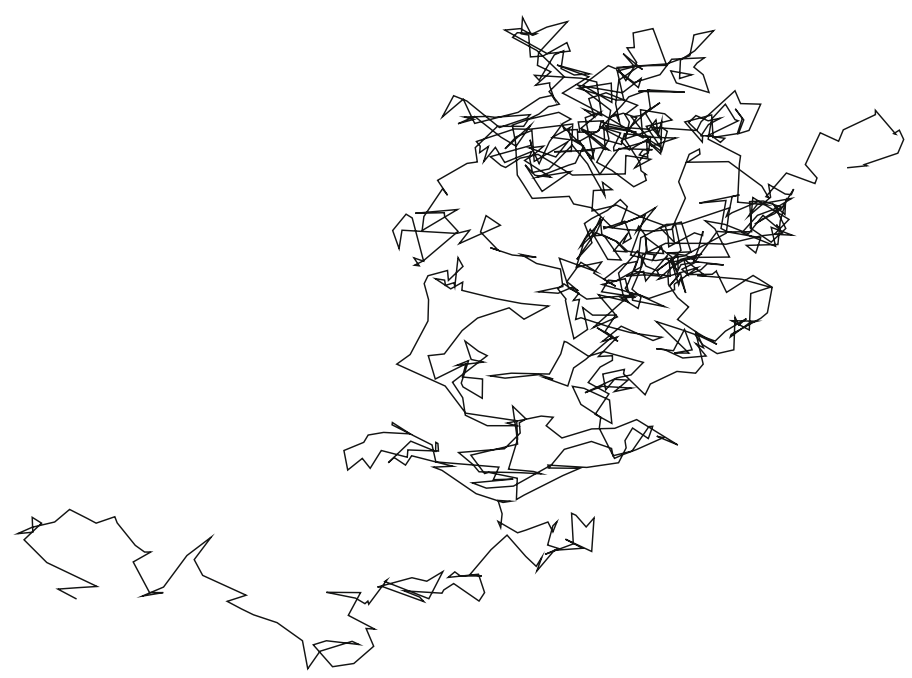

Fig. 1 A representative trajectory of a Brownian particle obtained by random walk particle tracking

\subsubsection{Brownian Motion}

Irregular motion of a particle in a fluid can be described by Newton's second law. The ambient fluid affects this motion in two opposite ways: The drag due to fluid viscosity slows the particle down, while the collisions with fluid molecules accelerate the particle (assuming the latter is sufficiently small). The drag force $F$ exerted by a fluid with dynamic viscosity $\mu$ on a (spherical) particle of radius $r_{p}$ is quantified by Stokes' law, $F=-6 \pi \mu r_{p}$. Figure 1 illustrates a typical trajectory of a Brownian particle.

The equations of motion of a particle moving along the one-dimensional $(d=1)$ trajectory $X(t)$ are

$$
\frac{\mathrm{d} X(t)}{\mathrm{dt}}=V(t), \quad \frac{\mathrm{d} V(t)}{\mathrm{dt}}=-\gamma V(t)+\sqrt{2 \kappa} \xi(t),
$$

where $\gamma=6 \pi \mu r_{p} / m$ is the drag coefficient, with $m$ denoting the particle's mass. The second term represents a random force that stands for the action of the surrounding liquid molecules. In the absence of the random forces, the particle velocity tends to 0 on the relaxation timescale $\tau_{\gamma}=\gamma^{-1}$. The random force $\xi(\mathrm{t})$ has zero mean and unit variance and correlated on a timescale much smaller than the relaxation time. Thus, the correlation function of $\xi(t)$ can be approximated as Gaussian white noise,

$$
\left\langle\xi(t) \xi\left(t^{\prime}\right)\right\rangle=\delta\left(t-t^{\prime}\right),
$$

where $\delta(t)$ is the Dirac delta. The angular brackets denote the average over all realizations of the random process $\xi(t)$.

The velocity process in (1) is an Ornstein-Uhlenbeck process (Risken 1996; Gardiner 2010). The parameter $\kappa$ is a priori unknown. However, it can be determined from the theorem of the equipartition of the kinetic energy between the degrees of freedom of a system in thermal equilibrium. The equipartition theorem implies that the velocity fluctuations in equilibrium, i.e., at times $t \gg \tau_{\gamma}$, are given by $\lim _{t \rightarrow \infty}\left\langle V(t)^{2}\right\rangle=k T / m$, where $k$ is the Boltzmann constant and $T$ is the absolute temperature. The angular brackets denote the average over all 
particles. At the same time, one obtains for the velocity fluctuations in equilibrium by solving for $V(t)$ in (1) the expression $\lim _{t \rightarrow \infty}\left\langle V(t)^{2}\right\rangle=\kappa / \gamma$. Thus, the strength of the random force is directly related to the dissipation of kinetic energy due to viscosity as

$$
\kappa=\frac{k T \gamma}{m} .
$$

This relation is an expression of the fluctuation-dissipation theorem (Kubo 1966). At times $t \gg \tau_{\gamma}$, the velocity $V(t)$ enters a dynamical steady state at which the fluctuations due to the random force are balanced by drag such that $v(t)=\sqrt{2 k T / m \gamma} \xi(t)$, where we used the expression (3) for $\kappa$. Using the definition of the drag coefficient $\gamma$ gives the velocity $V(t)=\sqrt{k T / 3 \pi \mu R}$, which is independent of the particle mass. The equation of motion of a particle for times $t \gg \tau_{\gamma}$ thus simplifies to

$$
\frac{\mathrm{d} X(t)}{\mathrm{d} t}=\sqrt{2 D_{0}} \xi(t), \quad D_{0}=\frac{k T}{m \gamma},
$$

where the coefficient $D_{0}$ is given by the Einstein-Smoluchowski relation. By using the above expression for $\gamma$, one obtains the Stokes-Einstein relation $D_{0}=k T /(6 \pi \mu R)$, which again is independent of the particle mass. The only particle property that enters into the diffusion coefficient is its size in terms of the radius $R$. The stochastic differential equation (4) can be seen as the starting point of the analyses of Einstein (1905) and von Smoluchowski (1906).

\subsubsection{The Diffusion Equation}

Let $f(x ; t)$ denote the probability density function (PDF) of finding a Brownian particle $X$ at point $x$ at time $t$. Our derivation of the evolution equation for $f(x, t)$ follows closely that in Einstein (1905). The stochastic process (4) constitutes a Markov process because a particle's position at a time $t+\Delta t$ depends solely on its position at the previous time $t$,

$$
X(t+\Delta t)=X(t)+w_{\Delta}, \quad w_{\Delta}=\sqrt{2 D_{0}} \int_{t}^{t+\Delta t} \xi\left(t^{\prime}\right) \mathrm{dt}^{\prime} .
$$

The variable $w_{\Delta}$ has a Gaussian PDF $f_{w}(x, t)$ with mean 0 and variance $2 D_{0} \Delta t$. The PDFs $f(x, t+\Delta)$ of $X(t+\Delta t)$ and $f(x, t)$ of $X(t)$ are related by

$$
f(x, t+\Delta)=\int_{-\infty}^{\infty} f\left(x-x^{\prime}, t\right) f_{w}\left(x^{\prime}, t\right) \mathrm{d} x^{\prime} .
$$

For small $\Delta t \ll t$, the variance of $f_{w}(x), 2 D_{0} \Delta t$, is small so that only small values of $x^{\prime}$ contribute to the integral. Hence, expanding $f(x, t+\Delta t)$ and $f\left(x-x^{\prime}, t\right)$ into respective Taylor series around the point $(x, t)$, we obtain an equation for $f(x, t)$ :

$$
\begin{aligned}
f+\frac{\partial f}{\partial t} \Delta t= & f+\int_{-\infty}^{\infty} f_{w}\left(x^{\prime}, t\right) \mathrm{d} x^{\prime}-\frac{\partial f}{\partial x} \int_{-\infty}^{\infty} x^{\prime} f_{w}\left(x^{\prime}, t\right) \mathrm{d} x^{\prime} \\
& +\frac{\partial^{2} f}{\partial x^{2}} \int_{-\infty}^{\infty} \frac{x^{\prime 2}}{2} f_{w}\left(x^{\prime}, t\right) \mathrm{d} x^{\prime}+\cdots
\end{aligned}
$$


Since the Gaussian PDF $f_{w}(x, \cdot)$ is symmetric, the second, fourth, etc., terms on the right side vanish, while the third, fifth, etc., are of order $\Delta t^{i}$ with $i=2,4$, etc. From the basic properties of a PDF, the first integral in (7) equals 1 , while the third integral is half of the variance, $\left(2 D_{0} \Delta t\right) / 2$. Therefore, (7) reduces to a diffusion equation

$$
\frac{\partial f(x, t)}{\partial t}=D_{0} \frac{\partial^{2} f(x, t)}{\partial x^{2}} .
$$

Thus, the coefficient $D_{0}$ defined in (4) is the diffusion coefficient.

As the particle motion is statistically isotropic and the motions along the coordinate axes are statistically independent, the above derivations can be readily generalized to $d$ spatial dimensions. If particles are injected at point $\mathbf{x}=\mathbf{0}$ of the $d$-dimensional infinite domain at time $t=0$, the initial PDF is $f(\mathbf{x}, t=0)=\delta(\mathbf{x})$ and the solution of the $d$-dimensional version of (8) is a Gaussian PDF

$$
f(\mathbf{x}, t)=\frac{1}{\left(4 \pi D_{0} t\right)^{d / 2}} \exp \left(-\frac{\mathbf{x}^{2}}{4 D_{0} t}\right) .
$$

Thus, the mean squared particle displacement along the $x$ coordinate axis until time $t$ is given by

$$
\left\langle X(t)^{2}\right\rangle \equiv \int_{-\infty}^{\infty} x^{2} f(x, t) \mathrm{dx}=2 D_{0} t .
$$

Using the expression for $D_{0}$ in (4) gives the following relation between the particle displacement and the particle size,

$$
r_{p}=\frac{k T t}{3 \pi \mu\left\langle X(t)^{2}\right\rangle} .
$$

Hence, the particle size can be obtained from the observation of the mean square displacement.

Another quantity of practical interest is the probability density of first particle arrival times at a given distance $x^{\prime}$ (Redner 2001). For simplicity, we consider the case of one-dimensional diffusion. For an initial position $X(t=0)=x^{\prime}$, we consider the distribution of first arrival times at the origin at $x=0$. The arrival time $T(x)$ is defined as

$$
T\left(x^{\prime}\right)=\min (t \mid X(t) \leq 0) .
$$

The first arrival time PDF $g\left(t, x^{\prime}\right)=\left\langle\delta\left[t-T\left(x^{\prime}\right)\right]\right\rangle$ can be determined in different ways. We choose here the following. The PDF $f\left(x^{\prime}, t\right)$ of finding a particle at $x=0$ at time $t$ that has started at $x^{\prime}$ at $t=0$ is

$$
f\left(x^{\prime}, t\right)=\int_{0}^{t} g\left(t^{\prime}, x^{\prime}\right) f\left(0, t-t^{\prime}\right) \mathrm{dt}^{\prime} .
$$

It is equal to the probability $g\left(t, x^{\prime}\right)$ that the particle arrives for the first time at the origin multiplied by the probability $f\left(0, t-t^{\prime}\right)$ that the particle returns to the origin after the time $t-t^{\prime}$ has passed. In Laplace space, the solution of this equation is

$$
\hat{g}\left(\lambda, x^{\prime}\right)=\frac{\hat{f}\left(x^{\prime}, \lambda\right)}{\hat{f}(0, \lambda)} .
$$




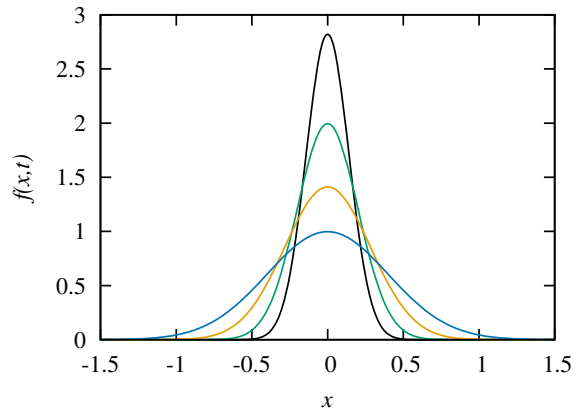

(a) particle density

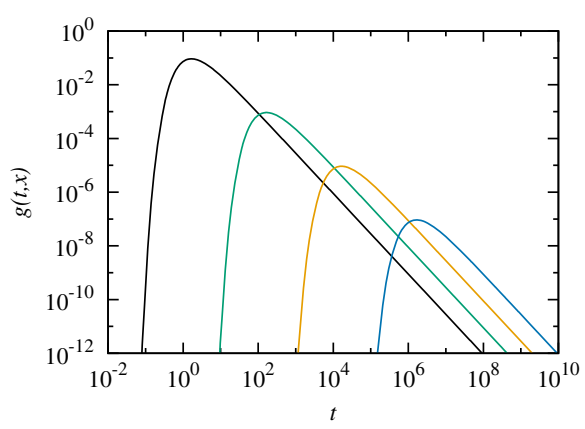

(b) arrival time density

Fig. 2 Illustration of a the probability density function $f(x, t)$ of particle positions $X(t)$ at (black, green, orange, blue) times $t=1,2,4,8 \cdot 10^{7} \mathrm{~s}$, and $\mathbf{b} g(t, x)$ of particle arrival times $T(x)$ at (black, green, orange, blue) $x^{\prime}=10^{-4}, 10^{-3}, 10^{-2}, 10^{-1} \mathrm{~m}$ for $D=10^{-9} \mathrm{~m}^{2} / \mathrm{s}$

The Laplace transform of $f$ in (9) is

$$
\hat{f}(x, \lambda)=\frac{1}{2 \sqrt{D \lambda}} \exp \left(-|x| \sqrt{\frac{\lambda}{D}}\right),
$$

so that the Laplace transform of the first arrival time PDF becomes

$$
\hat{g}\left(\lambda, x^{\prime}\right)=\exp \left(-\left|x^{\prime}\right| \sqrt{\frac{\lambda}{D}}\right),
$$

which is the Laplace transform of a Lévy-Smirnov density or inverse Gaussian density. Computing the inverse Laplace transform, we arrive at

$$
g\left(t, x^{\prime}\right)=\frac{x^{\prime}}{\sqrt{4 \pi D t^{3}}} \exp \left(-\frac{x^{\prime 2}}{4 D t}\right) .
$$

The Gaussian particle distribution and the inverse Gaussian first arrival time distribution are illustrated in Fig. 2.

\subsection{Diffusion of Finite-Size Particles}

Finite size of diffusing particles introduces two additional features: (i) such particles experience drag and hydrodynamic interactions while moving through a fluid, and (ii) the smallest distance between any two particles is limited by their radii, giving rise to exclusion volumes that the particles cannot enter. A macroscopic manifestation of these phenomena is the dependence of an effective diffusion coefficient $D$ on the particle concentration $c(\mathbf{x}, t)$ (e.g., Batchelor 1976; Bruna and Chapman 2012) and the references therein).

Consider a system of $N_{\text {par }}$ non-deformable spherical particles, whose radius $R$ is significantly smaller than a characteristic length $L$ of a simulation domain $\mathcal{D}$. At any given time $t$, the dynamics of the centroids of these particles, $\mathbf{X}_{i}(t)$ with $i=1, \ldots, N_{\text {par }}$, satisfies a stochastic differential equation

$$
\mathrm{d} \mathbf{X}_{i}=\sqrt{2 D_{0}} \mathrm{~d} \mathbf{B}_{i}+\mathbf{U d t}, \quad i=1, \ldots, N_{\mathrm{par}} .
$$


Here, $D_{0}$ is the coefficient of molecular diffusion defined by the kinetic theory of gases (Kennard 1938) in terms of a molecule's mean free path $\lambda$ and mean velocity $v$ as $D_{0}=\lambda v / 3$, $\mathbf{B}_{i}$ denotes the $d$-dimensional ( $d=2$ or 3 ) standard Brownian motion of the $i$ th particle, and $\mathbf{U}\left(\mathbf{X}_{i}\right)$ represents advection velocity or appropriately scaled external forces acting identically on all particles. The presence of the exclusion volumes implies that the random processes $\mathbf{X}_{i}(t)\left(i=1, \ldots, N_{\text {par }}\right)$ are no longer independent. The PDF, $f_{\mathbf{X}_{i}}(\mathbf{x}, t)$, of the random centroid $\mathbf{X}_{i}$ occupying the point $\mathbf{x}$ at time $t$ satisfies, up to first order in $\varepsilon=R / L$, a nonlinear partial differential equation (Bruna and Chapman 2012)

$$
\frac{\partial f_{\mathbf{X}_{i}}}{\partial t}=D_{0} \nabla_{\mathbf{x}}^{2}\left[f_{\mathbf{X}_{i}}+\alpha_{d}\left(N_{\mathrm{par}}-1\right) \varepsilon^{d} f_{\mathbf{X}_{i}}^{2}\right]-\nabla_{\mathbf{x}} \cdot\left[\mathbf{U}(\mathbf{x}) f_{\mathbf{X}_{i}}\right], \quad \mathbf{x} \in \mathcal{D} .
$$

Here, $\alpha_{d}=\pi / 2$ or $2 \pi / 3$ in $d=2$ or 3 spatial dimensions, respectively.

If the number of particles is large, such that $N_{\text {par }}-1 \approx N_{\text {par }}$, then it follows from (19) that their volumetric concentration, $c(\mathbf{x}, t) \equiv \varphi f_{\mathbf{X}_{i}}$ with $\varphi=\pi N_{\text {par }} \varepsilon^{d} /(2 d)$ denoting the volume fraction of particles relative to the volume of $\mathcal{D}$, satisfies a nonlinear advection-diffusion equation

$$
\frac{\partial c}{\partial t}=\nabla_{\mathbf{x}} \cdot\left[D(c) \nabla_{\mathbf{x}} c\right]-\nabla_{\mathbf{x}} \cdot[\mathbf{U}(\mathbf{x}) c], \quad \mathbf{x} \in \mathcal{D},
$$

with the concentration-dependent effective diffusion coefficient

$$
D(c)=D_{0}[1+4(d-1) c] .
$$

The finite particle size gives rise to a somewhat nontrivial collective behavior. First, the value of the effective diffusion coefficient $D$ depends on the dimensionality $d$. Second, $D$ differs from the "self-diffusion" coefficient of an individual particle, $D_{0}$; this is in contrast to point particles undergoing Brownian motion, for which these two diffusion coefficients are identical (Sect. 2.1). Third, (21) suggests that particles of the finite size diffuse faster (have higher effective diffusion coefficient, $D$ ) than their zero-volume counterparts $D_{0}$ (whose effective diffusion coefficient is $D_{0}$ ). That is because collisions of large particles introduce a bias to their random (Brownian) motion, thus accelerating their net spreading (Bruna and Chapman 2012).

\subsection{Diffusion of Electrically Charged Particles}

Consider Brownian motion (diffusion) of ions, which are treated as point charges. Diffusion coefficients of individual cations (the positively charged ions, denoted by the subscript "+") and anions (the negatively charged ions, denoted by the subscript "-") are denoted by $D_{+}$ and $D_{-}$, respectively. This Brownian motion of cations and anions in a solution results in spatial variability of their respective volumetric concentrations $c_{ \pm}(\mathbf{x}, t)$. The latter are related to the electrochemical potentials of cations and anions,

$$
\mu_{ \pm}(\mathbf{x}, t)=R T \ln c_{ \pm}(\mathbf{x}, t)+z_{ \pm} F \varphi(\mathbf{x}, t),
$$

where $z_{ \pm}$are the cation and anion charges (valencies); $R$ and $F$ are the gas and Faraday constants, respectively; $T$ is the temperature; and $\varphi(\mathbf{x}, t)$ is the electric potential. Spatial variability of $\mu_{ \pm}$induces ionic (Nernst-Planck) fluxes

$$
\mathbf{J}_{ \pm}^{\mathrm{NP}}=-M_{ \pm} c_{ \pm} \nabla \mu_{ \pm},
$$

where the ion mobility $M_{ \pm}$is related to the molecular diffusion coefficient of ions in the fluid, $D_{ \pm}$, by the Einstein relation $M_{ \pm}=D_{ \pm} / R T$. 
In the absence of homogeneous chemical reactions, mass conservation of anions and cations, $\partial_{t} c_{ \pm}=-\nabla \cdot \mathbf{J}_{ \pm}^{\mathrm{NP}}$, gives rise to the Nernst-Planck equations

$$
\frac{\partial c_{ \pm}}{\partial t}=\nabla \cdot\left(D_{ \pm} \nabla c_{ \pm}\right)-\nabla \cdot\left(\mathbf{U}_{ \pm} c_{ \pm}\right), \quad \mathbf{U}_{ \pm} \equiv-\frac{z_{ \pm} D_{ \pm} F}{R T} \nabla \varphi, \quad \mathbf{x} \in \mathcal{D} .
$$

The total (net) ionic charge density $q \equiv F\left(z_{+} c_{+}+z_{-} c_{-}\right)$is related to the electric potential $\varphi(\mathbf{x}, t)$ through a Poisson equation,

$$
-\mathcal{E} \nabla^{2} \varphi=F\left(z_{+} c_{+}+z_{-} c_{-}\right), \quad \mathbf{x} \in \mathcal{P},
$$

where $\mathcal{E}$ is dielectric constant of the solvent.

\subsection{Diffusion-Limited Chemical Reactions}

Chemical reactions are contact processes. They depend on the availability of reacting species and on the processes that bring them into contact, which here is diffusion. In this section, we briefly report three examples that illustrate the impact of diffusion on chemical reactions.

\subsubsection{Smoluchowski Theory}

To illustrate the main ideas behind the Smoluchowski reaction rate theory (von Smoluchowski 1917), we consider a fast irreversible bimolecular chemical reaction

$$
A+B \longrightarrow C \text {. }
$$

The species $A$ acts as a stationary sink, which is surrounded by $B$ particles at constant concentration $c_{B}$. The $A$ and $B$ particles annihilate when they are brought into contact; this means the intrinsic reaction kinetics are very fast. Diffusion is the rate-limiting process. The relative motion of the $A$ and $B$ particles is governed by the Langevin model discussed above. As $A$ and $B$ particles are idealized as point particles in the Langevin model for diffusion, we need to define a reaction or capture radius $r_{0}$. This means two particles are in contact and annihilate if their distance is smaller than $r_{0}$.

The relative distance $\mathbf{r}(t)=\mathbf{X}_{A}(t)-\mathbf{X}_{B}(t)$ between a particle $A$ and a particle $B$ satisfies the Langevin equation

$$
\frac{\mathrm{d} \mathbf{r}(t)}{\mathrm{dt}}=\sqrt{4 D_{0}} \boldsymbol{\xi}(t)
$$

Thus, the PDF $f(\mathbf{r}, t)$ of $\mathbf{r}(t)$ satisfies the diffusion equation (8) for the diffusion coefficient $2 D_{0}$. Under radial symmetry in $d=3$ dimensions, $f(\mathbf{r}, t) \rightarrow f(r, t)$ and

$$
\frac{\partial f}{\partial t}=\frac{2 D_{0}}{r^{2}} \frac{\partial}{\partial r}\left(r^{2} \frac{\partial f}{\partial r}\right) .
$$

In order to determine the reaction rate for a single $A$ particle, we consider the following boundary value problem. The concentration at $r=\infty$ is equal to $f=c_{B}$, and at $r=r_{0}$ we have an absorbing boundary such that $f\left(r_{0}, t\right)=0$. The steady-state solution $f_{\infty}(r)$ to (28)

$$
f_{\infty}(r)=c_{B}\left(1-\frac{r}{r_{0}}\right) \text {. }
$$

Thus, we obtain for the flux at $r=r_{0}$

$$
j_{B}=8 \pi D r_{0}^{2} \frac{\partial f_{\infty}}{\partial r}\left(r_{0}\right)=8 \pi D r_{0} c_{B}
$$


For the concentration $c_{A}$ of $A$ particles, this means that the reaction rate is

$$
R=8 \pi D r_{0} c_{A} c_{B}
$$

From this, it follows that the reaction rate constant is

$$
k=8 \pi D r_{0} .
$$

Thus, the reaction rate is determined by the diffusion rate.

\subsubsection{Ovchinnikov-Zeldovich Segregation}

Here, we briefly report on the impact of segregation due to heterogeneous initial reactant distributions in the presence of diffusive mass transfer (Ovchinnikov and Zeldovich 1978). For spatially uniform initial concentrations in a well-mixed reaction, the evolution of the concentrations $c_{i}$ of the $i=A, B, C$ species in the reaction (26) follows the kinetic rate law

$$
\frac{\mathrm{dc}_{i}(t)}{\mathrm{dt}}=-k c_{A}(t) c_{B}(t), \quad \frac{\mathrm{d} c_{C}(t)}{\mathrm{dt}}=k c_{A}(t) c_{B}(t)
$$

with $i=A, B$. For equal initial concentrations $c_{A}(t=0)=c_{B}(t=0)=c_{0}$, the solution for $c_{A}(t)=c_{B}(t)=c(t)$ is

$$
c(t)=\frac{c_{0}}{1+k c_{0} t} .
$$

Thus, for times larger than $1 / k c_{0}$, the reactant concentrations decay as $c(t) \sim 1 / t$.

Next, we consider the situation of heterogeneous initial distributions of the reacting species $A$ and $B$ such that the spatial averages $\bar{c}_{A 0}=\bar{c}_{B 0}$. The chemical reaction leads to a local depletion of the minority species and the formation of $A$ and $B$ islands, this means segregation of the reactants as illustrated in Fig. 3. Reactions between $A$ and $B$ particles are limited by diffusion to the island boundaries, where reactions can occur. For simplicity, we consider an instantaneous reaction such that $c_{A} c_{B}=0$. Furthermore, we assume that the fluctuations of initial particle numbers $\delta N_{0} \propto \sqrt{N_{0}}$. This implies that $\delta c_{A 0}=c_{A 0}-\bar{c}_{A 0}$ and $\delta c_{B 0}$ satisfy $\overline{V^{2} \delta c_{A 0}{ }^{2}}=\overline{V^{2} \delta c_{B 0}{ }^{2}}=c_{A 0} V$, where $V$ is the volume of an island. The difference $u=c_{A}-c_{B}$ is conservative and subject to diffusion only. The mean and variance of its initial distribution $u_{0}$ are $\bar{u}_{0}=0$ and $\overline{V^{2} u_{0}^{2}}=2 c_{A 0} V$. The $A$ islands, characterized by $u>0$, and the $B$ islands, characterized by $u<0$, grow diffusively. This means their typical size grows as $\ell \sim \sqrt{D t}$. Thus, in an island of volume $V \sim(D t)^{d / 2}$, the typical value

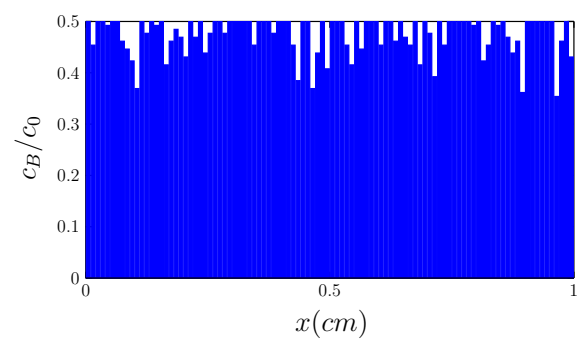

(a) initial distribution

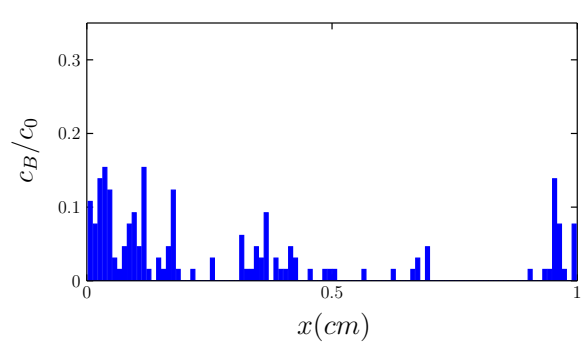

(b) island formation

Fig. 3 Island formation in an instantaneous irreversible bimolecular reaction under heterogeneous initial conditions in one spatial dimension 
of $u$ is given in terms of the initial excess of the majority species per volume; this means $|u| \sim\left|\delta N_{0}\right| / V \sim \sqrt{c_{A 0} V} / V \sim V^{-1 / 2}$. As locally $|u|=c_{A}$ or $c_{B}$, we obtain

$$
\bar{c}_{A}(t) \sim \frac{1}{(D t)^{d / 4}} .
$$

This behavior is valid for $d<3$. The segregation of the reacting species due to the heterogeneous initial distribution significantly slows down the reaction kinetics relative to the well-mixed case. The detailed analysis of Ovchinnikov-Zeldovich segregation using spectral analysis and numerical simulations can be found in Toussaint and Wilczek (1983), Kang and Redner (1985), among many others.

\subsubsection{Diffusion in the Presence of Randomly Distributed Traps}

An illustrative example for the impact of chemical heterogeneity on reactivity is diffusion in a medium characterized by a random distribution of traps, which are characterized by a constant number density $n_{0}$. The trap positions are distributed uniformly, and the traps are allowed to overlap (Kayser and Hubbard 1983). The random distribution of traps may be identified with a distribution of specific reactive surface area in porous media. The concentration $c_{A}(\mathbf{x}, t)$ of a species $A$ evolves according to the diffusion equation $\partial_{t} c_{A}=D \nabla^{2} c_{A}$. At the surface of each trap, absorbing boundary conditions are specified, $c_{A}(\mathbf{x}, t)=0$.

For $d=1$ spatial dimension, the distance $\ell$ between traps is distributed exponentially as $\sim \exp \left(-n_{0} \ell\right)$. The average concentration of species $A$ between two traps behaves at asymptotically long times $t \gg \ell^{2} / D$ as (Havlin and Ben-Avraham 2002)

$$
\bar{c}_{A}(t, \ell)=\frac{1}{\ell} \int_{0}^{\ell} c_{A}(x, t) \mathrm{dx} \propto \exp \left(-\pi^{2} D t / \ell^{2}\right) .
$$

The overall average concentration of species $A$ is obtained by spatially averaging over all trap-free intervals such that (Kayser and Hubbard 1983)

$$
\bar{c}_{A}(t)=\int_{0}^{\infty} n_{0}^{2} \ell \exp \left(-n_{0} \ell\right) \bar{c}_{A}(t, \ell) \mathrm{d} \ell \sim \exp \left[-\gamma\left(t / \tau_{D}\right)^{1 / 3}\right],
$$

where $\gamma$ is a dimensionless constant and $\tau_{D}=n_{0}^{2} D$ is the characteristic diffusion time between traps. For higher dimensions, a similar reasoning gives

$$
\bar{c}_{A}(t) \gtrsim \exp \left[-\gamma\left(t / \tau_{D}\right)^{d /(d+2)}\right] .
$$

The overall reaction kinetics is slower than what would be predicted by a first-order decay, $\bar{c}_{A}(t) \sim \exp \left(-t / \tau_{D}\right)$, based on a mean decay time equal to $\tau_{D}$, the diffusion time between traps, which would be a reasonable first guess for the reactivity of the system. This behavior is due to the fact that there is a small but finite probability of finding intervals or holes that are arbitrarily large with an associated very large diffusion time and thus survival time. Figure 4 illustrates the evolution of the species concentrations according to the exponential and stretched exponential laws. 
Fig. 4 Evolution of the species concentration under randomly distributed traps in $d=1$ (blue), $d=2$ (orange), and $d=3$ (green) spatial dimensions for $\gamma=1$. The black line denotes the exponential decay law

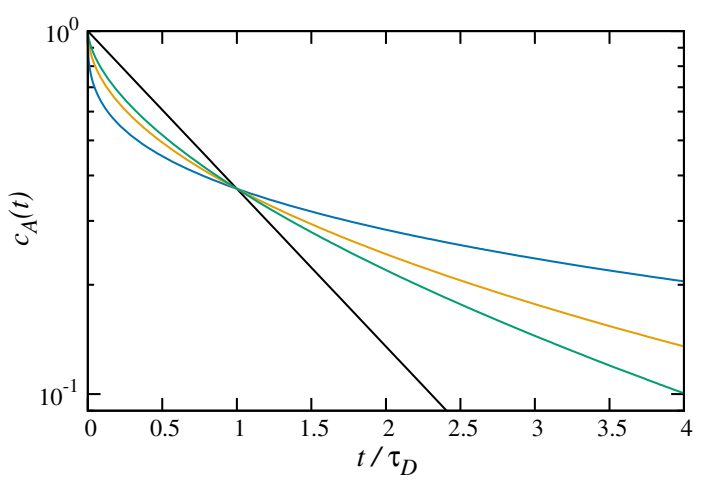

\section{Molecular Diffusion in Crowded Environments}

\subsection{Geometrically Constrained Diffusion}

The diffusion models described above assume that the individual and/or collective motion of particles is unaffected by the geometry of a simulation domain (e.g., pore space) $\mathcal{D}$. This assumption can be violated when pores become small, in extreme cases reaching the size of diffusing particles (e.g., red blood cells moving through capillaries). If particles move in a $d$-dimensional $(d=2$ or 3 ) space $\mathcal{D}$ whose characteristic size $w$ in $k(k<d)$ dimensions is exceedingly small, it is common to introduce diffusion models in an "effective dimension" $d_{\text {eff }}=d-k$. For example, the effective dimension of a transport model in a narrow long capillary $(d=2)$ is $d_{\text {eff }}=1$, while that in a narrow fracture is $d_{\text {eff }}=2$.

Under such conditions, (19) is replaced with a PDF equation (Bruna and Chapman 2014),

$$
\frac{\partial f_{\mathbf{X}_{i}}}{\partial t}=D_{0} \nabla_{\mathbf{x}}^{2}\left[f_{\mathbf{X}_{i}}+\alpha_{h}\left(N_{\text {par }}-1\right) \varepsilon^{d_{\text {eff }}} f_{\mathbf{X}_{i}}^{2}\right]-\nabla_{\mathbf{x}} \cdot\left[\overline{\mathbf{U}}(\mathbf{x}) f_{\mathbf{X}_{i}}\right], \quad \mathbf{x} \in \mathcal{D}_{\text {eff }},
$$

which is defined in a domain $\mathcal{D}_{\text {eff }}$ with the effective dimension $d_{\text {eff }}$. In special cases, the coefficient $\alpha_{h}$ can be related analytically to the "confinement parameter" $h$ (Bruna and Chapman 2014); otherwise, it serves as a fitting parameter. Equation (39) governs the PDF of finding the $i$ th finite-size particle, $f_{\mathbf{X}_{i}}(\mathbf{x}, t)$, at the space-time point $(\mathbf{x}, t)$.

Collective diffusion of a large number of finite-size particles, $N_{\text {par }}-1 \approx N_{\text {par }}$, is described by the nonlinear advection-diffusion equation (20) with the following caveats. First, the volume fraction $\varphi_{h}$ in the definition of the volumetric concentration $c \equiv \varphi_{h} f_{\mathbf{X}_{i}}$ can now be explicitly derived only in a few special cases for which $\alpha_{h}$ is computable (Bruna and Chapman 2014). Second, (20) is defined in the domain $\mathcal{D}_{\text {eff }}$ of the effective (reduced) dimension $d_{\text {eff }}$; the drift velocity vector $\overline{\mathbf{U}}$ has the corresponding number of components. Third, the effective diffusion coefficient $D(c)$ in (21) is replaced with

$$
D(c)=D_{0}\left[1+g_{h} c\right],
$$

wherein the coefficient $g_{h}>0$ is explicitly given for the same special cases.

For point particles, both (39) and the corresponding nonlinear equation for the particle concentration reduce to a linear $\mathrm{ADE}$

$$
\frac{\partial \mathcal{A}}{\partial t}=D_{0} \nabla_{\mathbf{x}}^{2} \mathcal{A}-\nabla_{\mathbf{x}} \cdot[\overline{\mathbf{U}}(\mathbf{x}) \mathcal{A}], \quad \mathbf{x} \in \mathcal{D}_{\text {eff }} .
$$


Here, $\mathcal{A}$ stands for both the PDF $f_{\mathbf{X}_{i}}(\mathbf{x}, t)$ and the concentration $c(\mathbf{x}, t)$. Since the particles have zero diameter, the "smallness" of $\mathcal{D}$ is expressed in terms of the Knudsen number $\mathrm{Kn}=\lambda / w$, where $\lambda$ is the mean free path of a particle diffusing in the space with the characteristic length $w$. Two special cases of the general, yet approximate, Eq. (41) are described below.

\subsubsection{Knudsen Diffusion}

If advection-diffusion transport in a pore $(\mathcal{D})$ of diameter $w$ takes place in the regime with $\mathrm{Kn} \ll 1$, then the pore is sufficiently large for the confinement effects to be negligible and the diffusion coefficient is $D=D_{0} \equiv \lambda v / 3$ where $v$ is the mean molecular velocity. If $\mathrm{Kn} \gg 1$, then the (Knudsen) diffusion coefficient is $D=D_{\mathrm{Kn}} \equiv w v / 3$ (Jacobs 1967). In the intermediate regime, the Bosanquet relation estimates the effective diffusion coefficient $D$ as the harmonic mean between $D_{0}$ and $D_{\mathrm{Kn}}$ (e.g., Zalc et al. 2004),

$$
D=\left(\frac{1}{D_{0}}+\frac{1}{D_{\mathrm{Kn}}}\right)^{-1} \text {. }
$$

This empirical treatment of diffusion replaces (41) with an ADE

$$
\frac{\partial c}{\partial t}=D \nabla_{\mathbf{x}}^{2} c-\nabla_{\mathbf{x}} \cdot[\mathbf{U}(\mathbf{x}) c], \quad \mathbf{x} \in \mathcal{D},
$$

i.e., allows one to obtain the concentration $c(\mathbf{x}, t)$ by solving the standard ADE, albeit with the modified diffusion coefficient.

Knudsen diffusion plays an important role in a large number of natural and engineered (nano)porous media. Examples include transport of various species in pharmaceutical tablets (Klinzing and Zavaliangos 2016), in tubular sublimators utilized for purification of large organic molecules (Qian et al. 2016), in graphites designed for high-temperature gas-cooled nuclear reactors (Kane et al. 2018), in membranes used for distillation desalination (Deshmukh and Elimelech 2017), and in hierarchical metamaterials tailored for energy storage devices (Zhang and Tartakovsky 2017).

\subsubsection{Fick-Jacobs Diffusion}

Consider advection-diffusion transport in a two-dimensional $(d=2)$ channel of a small width $w=H$, with the transverse coordinate $-H / 2<y<H / 2$. The corresponding effective dimension is $d_{\text {eff }}=1$; the resulting effective model is defined on the domain $D_{\text {eff }}=\{x:-L<x<L\}$, with the confinement parameter $h=H / \varepsilon$. This model takes the form of a one-dimensional version of (41),

$$
\frac{\partial \mathcal{A}}{\partial t}=D_{0} \frac{\partial^{2} \mathcal{A}}{\partial x^{2}}-U \frac{\partial \mathcal{A}}{\partial x}, \quad-L<x<L .
$$

Here, $\mathcal{A}(x, t)$ stands for both the PDF $f_{X_{i}}(x, t)$ and the concentration $c(x, t)$. A generalization of (44), which accounts for spatial variability of the channel width, $h=h(x)$, is referred to as the Fick-Jacobs equation (Jacobs 1967; Bruna and Chapman 2014),

$$
\frac{\partial \mathcal{A}}{\partial t}=D_{0} \frac{\partial}{\partial x}\left[h \frac{\partial}{\partial x}\left(\frac{\mathcal{A}}{h}\right)\right]-U \frac{\partial \mathcal{A}}{\partial x}, \quad-L<x<L .
$$

This equation and its multiple variants (see, e.g., Dorfman and Yariv (2014) and Sect. 4 in Burada et al. (2009)) have been used to describe pore-scale diffusion processes in biological 
(e.g., ion channels), geological (e.g., tight formations), and manufactured (e.g., nanotubes) porous media.

\subsection{Continuum (Darcy-Scale) Representations of Diffusion in Porous Media}

The diffusion models described in the previous sections are valid for the fluid phase (pore space) of a porous medium. Consequently, they can be deployed in pore-scale simulations of transport processes. Their use at the continuum (Darcy) scale requires either systematic upscaling (see, e.g., Battiato et al. (2019) in this issue for a comparative review of various upscaling techniques) or empirical modifications.

For example, a commonly used (and often criticized) expression,

$$
D_{\text {eff }}=\frac{\omega D}{\tau},
$$

relies on geometric characteristics of a porous medium-its porosity $\omega$ and tortuosity $\tau$ - to relate the Darcy-scale diffusion coefficient $D_{\text {eff }}$ to its pore-scale counterpart $D$. Combining (46) with the (empirical) Bruggeman relation $\tau=1 / \sqrt{\omega}$ yields another popular model, $D_{\text {eff }}=\omega^{3 / 2} D$. The Darcy-scale transport models of this kind assume that the pore- and Darcy-scale equations, such as those described above, are identical except for the values of their diffusion coefficients.

Even for linear diffusion phenomena, empirical relations such as $D_{\text {eff }}=\omega^{3 / 2} D$ or (46) fail to capture many salient features of the Darcy-scale diffusivity, including its tensorial nature due to pore geometry (Battiato et al. 2019). For nonlinear phenomena, e.g., diffusion of charged particles (Sect. 2.3), these relations do not account for the observed dependence of $D_{\text {eff }}$ on solute/electrolyte properties and transport conditions (e.g., the electrical double layer that forms on the solid surfaces, thus reducing the pore space available for diffusion) (Zhang and Tartakovsky 2017). Even if the empirical models like (46) were sufficiently accurate, they are of limited use in materials design that aims to identify an optimal pore geometry (Zhang et al. 2015).

In a somewhat tautological way, (46) can be used as a definition of a porous medium's tortuosity $\tau$. It allows one to estimate a value of the otherwise unobservable $\tau$ from measurements of $D$ and $D_{\text {eff }}$. While the thermodynamic considerations yield a value of the pore-scale (free fluid) diffusivity $D$ for many of the diffusion phenomena described above, the value of $D_{\text {eff }}$ has to be inferred by fitting a solution of, e.g., the ADE (43) to Darcy-scale concentration measurements. The latter step assumes both the validity of ADE-like representations of Darcy-scale diffusion phenomena and the dependence of $D_{\text {eff }}$ solely on geometric properties of the pore space. Since both assumptions are problematic (see the discussion above and Sect. 5), this procedure for estimating the tortuosity can lead to unphysical results. For example, "tortuosity factors are often much larger for Knudsen diffusion than for bulk diffusion, in spite of their intended and purely geometric nature" (Zalc et al. 2004).

\subsection{Trap and Symmetric Barrier Models}

For diffusion in a fluid at rest, the density of particles which move according to the Langevin equation (4) is described by the diffusion equation (8). The latter is a combination of Fick's law of diffusion, $J=-D \partial_{x} f$, and mass conservation, $\partial_{t} f=-\partial_{x} J$. For diffusion in heterogeneous media, one distinguishes between the symmetric barrier and trap models 
Fig. 5 Steady-state particle distributions for the trap (green) and symmetric (black) barrier models. The blue line denotes the diffusion coefficient $D(x)$

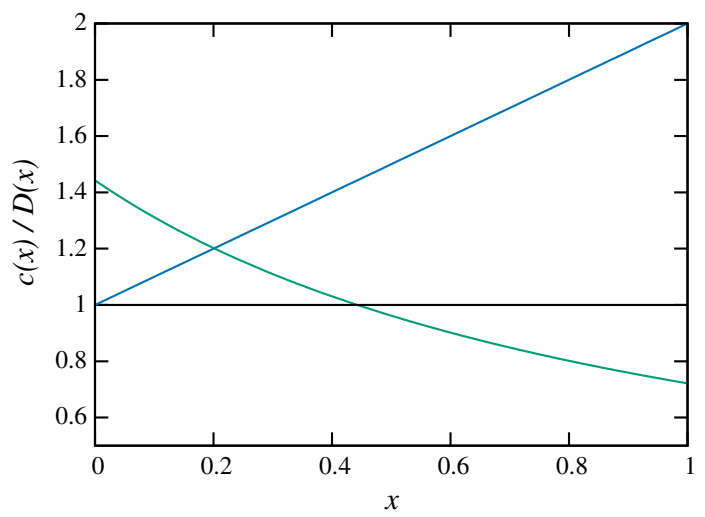

(Bouchaud and Georges 1990). These alternative models and their implications are discussed below.

\subsubsection{Trap Model}

The Langevin equation (4) with a spatially variable diffusion coefficient,

$$
\frac{\mathrm{d} X(t)}{\mathrm{dt}}=\sqrt{2 D[X(t)]} \xi(t),
$$

gives rise to a Fokker-Planck equation for the PDF $f(x, t)$ (Risken 1996; Gardiner 2010),

$$
\frac{\partial f}{\partial t}=\frac{\partial^{2} D(x) f}{\partial x^{2}} .
$$

Its steady-state solution, the $\mathrm{PDF} f^{\mathrm{eq}}(x) \propto 1 / D(x)$, implies that particles accumulate in regions of small diffusion coefficient $D(x)$, i.e., the particles get trapped. Figure 5 shows the steady-state particle distribution for a linearly varying $D(x)=x+1$ in a domain $0<x<1$ with reflecting boundary conditions. Particles accumulate, i.e., are trapped where the diffusion coefficient is small.

\subsubsection{Symmetric Barrier Model}

An alternative way to account for heterogeneity is to generalize Fick's law, $J=-D(x) \partial_{x} f$. Combined with mass conservation, this yields a diffusion equation for $f(x, t)$,

$$
\frac{\partial f}{\partial t}=\frac{\partial}{\partial x}\left[D(x) \frac{\partial f}{\partial x}\right] \text {. }
$$

Its steady-state solution, the PDF $f^{\mathrm{eq}}=$ constant, implies that particles are uniformly distributed, i.e., there is no accumulation of mass in regions of high or low diffusivity. The Langevin equation corresponding to (49) is (Risken 1996; Gardiner 2010)

$$
\frac{\mathrm{d} X(t)}{\mathrm{dt}}=\frac{\mathrm{d} D[X(t)]}{\mathrm{dx}}+\sqrt{2 D[X(t)]} \xi(t) .
$$

The drift term drives the particles away from regions of low diffusivity toward those of high diffusivity. This drift counteracts the trapping effect of Sect. 3.3.1 and thus leads to a uniform particle distribution. 
The term "symmetric barrier model" is illustrated by a finite-volume discretization of (49),

$$
\frac{\mathrm{d} f_{n}(t)}{\mathrm{d} t}=\sum_{m} w_{n m}\left[f_{m}(t)-f_{n}(t)\right], \quad f_{n}(t) \equiv f\left(x_{n}, t\right) .
$$

Equation (51) is a master equation with the transition rates

$$
w_{n m}=\frac{\hat{D}_{n m}}{\Delta x^{2}},
$$

where $\hat{D}_{n m}$ is the bond diffusivity between cells $n$ and $m$, which is typically determined as the harmonic mean between the diffusion coefficient in the neighboring cells. The symmetry of the transition rates, $w_{m n}=w_{n m}$, gives rise to the term "symmetric barrier model."

\section{Diffusion-Like Phenomena in Porous Media}

Linear and nonlinear diffusion equations provide Darcy-scale representations of various flow and transport phenomena in porous media that are related to the phenomenon of diffusion in a wider sense. These phenomena are not necessarily related to the motion of Brownian particles in a free fluid, but describe instead diffusion of pressure and diffusive propagation of a phase field such as fluid saturation. We also discuss diffusion-like phenomena of solute dispersion that are caused by hydrodynamic velocity fluctuations.

\subsection{Flow in Porous Media}

Linear diffusion: single-phase flows. Consider a porous medium that is completely saturated with a fluid of density $\rho$ and dynamic viscosity $\mu$. Within the pore space, fluid flow can be described by Stokes or Navier-Stokes equations, depending on the pore size and flow velocity. When averaged over a sufficiently large volume of the medium, i.e., at the Darcy scale, the same flow is described by Darcy's law $\mathbf{q}=-K \nabla h$ which, in analogy with Fick's law of diffusion, postulates a linear relation between the fluid's volumetric (Darcy) flux $\mathbf{q}$ and the gradient of hydraulic head $h=\psi-x_{3}$. Here, $\psi=p /(\rho g)$ is the pressure head, with $g$ denoting the gravitational acceleration constant; and the vertical coordinate $x_{3}$ (positive downward) represents the elevation head. The constant of proportionality $K=\mathrm{k} \rho \mathrm{g} / \mu$ in Darcy's law is called hydraulic conductivity; it is a property of both the porous medium (via its dependence on $k$, the medium's permeability) and the fluid (its density $\rho$ and viscosity $\mu$ ). Darcy's law is essentially phenomenological, even though it can be derived (after many approximations) from the pore-scale Stokes equations by means of homogenization (Battiato et al. 2019).

When combined with mass conservation for a fluid-saturated volume of the porous medium, $\partial_{t}(\omega \rho)=-\nabla \cdot \mathbf{q}$, Darcy's law yields a diffusion equation for the hydraulic head $h(\mathbf{x}, t)$,

$$
\frac{\partial h}{\partial t}=D \nabla^{2} h,
$$

where $D \equiv K / S_{S}$ is the water diffusivity and the specific storage $S_{S}$ accounts for slight compressibility of both the fluid and the porous matrix. This equation is written for homogeneous and isotropic porous media and implies the absence of fluid sources and sinks. Its generalizations, which account for these features, are straightforward. 
Nonlinear diffusion: multiphase flows. Consider next a porous medium whose pores are filled with air and water. The fraction of the pores occupied by water, in a (representative elementary) volume of the medium, is referred to as water saturation, $S_{w} \leq 1$. A Darcy-scale description of water flow through partially saturated porous media relies on a phenomenological generalization of the phenomenological Darcy's law, $\mathbf{q}=-K\left(S_{w}\right) \nabla h$, in which the hydraulic conductivity is an increasing function of saturation. Its relationship to, and derivation from, the pore-scale Stokes equations for multiple fluids separated by immiscible interfaces is even more tenuous than that for single-phase flows (Battiato et al. 2019). That is, in part, because the hydraulic conductivity $K$ is affected not only by the saturation $S_{w}$ but also by the fluid topology (Picchi and Battiato 2018; Picchi et al. 2018).

Assuming the air pressure throughout the porous medium to equilibrate (nearly) instantaneously with the atmosphere, and combining the Darcy's law with mass conservation, $\omega \partial_{t} S=-\nabla \cdot \mathbf{q}$ leads to the Richards equation written in terms of the water content $\theta(\mathbf{x}, t) \equiv \omega S(\mathbf{x}, t)$,

$$
\frac{\partial \theta}{\partial t}=\nabla \cdot[D(\theta) \nabla \theta]-U(\theta) \frac{\partial \theta}{\partial x_{3}} .
$$

The moisture diffusivity $D(\theta) \equiv K(\theta)(\mathrm{d} \psi / \mathrm{d} \theta)$ and the "velocity" $U(\theta) \equiv \mathrm{d} K / \mathrm{d} \theta$ are calculated from the experimentally determined constitutive relations $\theta=\theta(\psi)$ and $K=$ $K(\theta)$.

Unlike the $\psi$-based Richards equation, the nonlinear ADE (54) cannot be used in fully saturated regions of a porous medium and its solutions for composite porous materials exhibit jump discontinuities. Generalizations of the Richards equation include systems of coupled nonlinear parabolic equations of multiphase flow, which track spatiotemporal evolution of the saturation (and pressure) of each fluid phase; and phase-field dynamic models of wetting and drying of porous media (Mitkov et al. 1998), which capture the observed hysteresis in the relative conductivity $K=K(\psi)$.

\subsection{Dispersion}

\subsubsection{Hydrodynamic Dispersion}

Upscaling of transport equations from the pore to the Darcy scale is the subject matter of Battiato et al. (2019) in this issue. Under a number of assumptions, this procedure leads to what is otherwise a phenomenological advection-dispersion equation for the Darcy-scale solute concentration $c(\mathbf{x}, t)$,

$$
\omega \frac{\partial c}{\partial t}=\nabla \cdot(\mathbf{D} \nabla c)-\nabla \cdot(\mathbf{q} c)
$$

The hydrodynamic dispersion tensor $\mathbf{D}$ accounts for both pore-scale molecular diffusion $D$ and spatial variability of pore-scale fluid velocity. For pore geometry with a characteristic length (e.g., average grain size) $\ell_{g}$, one defines a Péclet number as Pe $=\bar{u} \ell_{g} / D$, where $\bar{u}=$ $|\overline{\mathbf{q}}| / \omega$ is the magnitude of the average pore velocity. For high Péclet numbers, the correlation time in the mean flow direction is $\tau_{u} \approx \ell_{g} / \bar{u}$ and the velocity variance $\sigma_{u}^{2} \sim \bar{u}^{2}$. Thus, the effect of velocity fluctuations on the longitudinal dispersion (the $D_{11}$ component of the dispersion tensor $\mathbf{D}$ when the $x_{1}$ coordinate is aligned with the flow direction) is

$$
D_{L} \sim \bar{u} \ell_{g} .
$$


This implies that $D_{L} / D \sim \mathrm{Pe}$, which was observed in experiments and numerical simulations at high Péclet numbers (Pfannkuch 1963; Bear 1972; Bijeljic and Blunt 2006). For $\mathrm{Pe}<\mathrm{Pe}_{\mathrm{cr}}$ with the critical Péclet number $\mathrm{Pe}_{c}=400$, the longitudinal dispersion coefficient scales as $D_{L} / D \sim \mathrm{Pe}^{1.2}$, and for $\mathrm{Pe} \ll 1$ as $D_{L} / D \sim 1$ (Bijeljic and Blunt 2006). These behaviors are captured by the relation (Bear 1972)

$$
D_{L}=D \gamma+\alpha_{L} \bar{u} \frac{\mathrm{Pe}}{\mathrm{Pe}+2+4 \delta^{2}},
$$

where $\alpha_{L}$ is the longitudinal dispersion length, which corresponds to the grain size $\ell_{g} ; \gamma$ represents the effect of the tortuous pore geometry on molecular diffusion in the bulk, e.g., $\gamma=\omega / \tau$ in (46); and the parameter $\delta$ characterizes the shape of the pore channels. The second term on the right side of (57) is mechanical dispersion.

\subsubsection{Macrodispersion}

Spatial variability of hydraulic conductivity $K(\mathbf{x})$ at the field scale is modeled by representing its logarithm, $Y=\ln K$, as a multivariate Gaussian random field, whose statistics (the mean $\bar{Y}$, variance $\sigma_{Y}^{2}$, and correlation length $\ell_{Y}$ ) are inferred from measurements. Upscaling of transport equations from the Darcy to the field scale by means of stochastic perturbation theory (Cushman 1997; Dagan 2012; Dagan and Neuman 1997) gives, under several approximations, an advection-dispersion equation (55) with the dispersion coefficient $\mathbf{D}$ and Darcy velocity $\mathbf{q}$ replaced by their effective counterparts $\mathbf{D}^{*}$ and $\overline{\mathbf{q}}$. The Péclet number is now defined as $\mathrm{Pe}=\bar{u} \ell_{Y} / D_{L}$, where $D_{L}$ in this context represents the (constant) local scale dispersion and $\bar{u}=|\overline{\mathbf{q}}| / \omega$ is the average macroscopic flow velocity magnitude. Again, at high Péclet numbers the correlation time is estimated as $\tau_{u}=\ell_{Y} / \bar{u}$. The first-order (in $\sigma_{Y}^{2}$ ) perturbation theory estimates the velocity variance to be $\sigma_{u}^{2} \sim \sigma_{Y}^{2} \bar{u}^{2}$ (e.g., Dagan 1984). Thus, the longitudinal macrodispersion coefficient is (e.g., Dagan 1984)

$$
D_{L}^{*}=\sigma_{Y}^{2} \bar{u} \ell_{Y} .
$$

This result relates the field-scale dispersion coefficient to the medium's hydraulic properties $\left(\sigma_{Y}^{2}\right.$ and $\left.\ell_{Y}\right)$ and the mean flow velocity $(\bar{u})$.

\section{Non-Fickian Diffusion in Porous Media}

Fickian diffusion (and dispersion) models predict the first and second centered moments of a solute plume (i.e., its center of mass and the spread) to increase linearly with time, $\left\langle X(t)^{2}\right\rangle \propto t$; the spatial distribution of a solute plume to have a Gaussian shape (9); and the solute breakthrough curves to be inversely Gaussian. A number of factors, e.g., the finite size of particles diffusing in confined environments (Sects. 2.2 and 3.1) or the electrical charge carried by particles (Sect. 2.3), can cause a deviation from this behavior; in other words, one could argue that the Fickian behavior is more rare ("anomalous") than the non-Fickian one (Cushman and O'Malley 2015).

Heterogeneity of porous media is another factor that gives rise to non-Fickian dynamics. Breakthrough curves are characterized by strong tailing, plumes exhibit non-Gaussian shapes with pronounced forward and/or backward tails, and both a plume (Bouchaud and Georges 1990; Cushman et al. 2009) and a particle's trajectory (Regner et al. 2013) grow nonlinearly in time, 


$$
\left\langle X(t)^{2}\right\rangle \propto t^{\alpha}, \quad 0<\alpha \leq 2,
$$

with the values of the exponent $0<\alpha<1$ and $1<\alpha \leq 2$ indicating the sub- and superdiffusive behavior, respectively. Inferring the exponent's value from (typically) noisy data is not straightforward (Cushman and Moroni 2001; Moroni and Cushman 2001; Regner et al. 2013, 2014); renormalization group classification methods (O'Malley and Cushman 2012; O'Malley et al. 2014) proved to be robust even for data with high noise-to-signal ratios (Regner et al. 2014).

While differing in their foundational assumptions and data requirements, models of nonFickian transport in heterogeneous media are interrelated in the sense that all are nonlocal in space and/or time, i.e., the (advection-)diffusion equation are replaced with their integrodifferential counterparts (Neuman and Tartakovsky 2009). The latter reference provides a plethora of comparative studies that focus on the commonalities, differences, and relative strengths of the competing models of non-Fickian transport. A brief description of a few of representative models is provided below.

\subsection{Ensemble-Averaged Nonlocal Diffusion Equations}

Solving a $d$-dimensional $(d \geq 1)$ version of the diffusion equation (49) with spatially varying $D(\mathbf{x})$ would yield $f(\mathbf{x}, t)$ that differs from the Gaussian distribution (9), which is obtained for the constant $D=D_{0}$. Hence, even though that equation follows from Fick's law, $\mathbf{q}=$ $-D(\mathbf{x}) \nabla f$, it is common to refer to such diffusion as non-Fickian. That is because, while written in the differential (infinitesimal) form, every term in Fick's law has a support scale $\mathcal{S}_{\text {sup }}$ on which it is measured and the spatial variability of, for example, $D(\mathbf{x})$, on scales smaller than $\mathcal{S}_{\text {sup }}$ is unresolved.

Within the probabilistic framework (Cushman 1997; Dagan 2012; Dagan and Neuman 1997), this lack of information about the sub-scale variability of $D$ is handled by treating it as a random field so that the corresponding diffusion equation, e.g., $d$-dimensional version of (49) defined on the computational domain $\mathcal{D} \in \mathbb{R}^{d}$, becomes stochastic. Ensemble-averaging of this equation yields (under some approximations) a space-time nonlocal (integro-differential) equation for the mean state variable, $\bar{f}(\mathbf{x}, t)$ (Neuman et al. 1996):

$$
\frac{\partial \bar{f}}{\partial t}=\nabla \cdot[\bar{D} \nabla \bar{f}]-\nabla \cdot \int_{0}^{t} \int_{\mathcal{D}} \kappa\left(\mathbf{x}, \mathbf{x}^{\prime}, t-t^{\prime}\right) \nabla \bar{f}\left(\mathbf{x}^{\prime}, t^{\prime}\right) \mathrm{d} \mathbf{x}^{\prime} \mathrm{dt}^{\prime} .
$$

The kernel $\kappa\left(\mathbf{x}, \mathbf{x}^{\prime}, t-t^{\prime}\right) \approx C_{D}\left(\mathbf{x}, \mathbf{x}^{\prime}\right) \nabla_{\mathbf{x}} \nabla_{\mathbf{x}^{\prime}}^{\top} G\left(\mathbf{x}, \mathbf{x}^{\prime}, t-t^{\prime}\right)$ is a symmetric positivesemidefinite second-rank tensor (dyadic) that is related to the two-point covariance function of the random diffusion coefficient, $C_{D}\left(\mathbf{x}, \mathbf{x}^{\prime}\right)$, and the mean-field Green's function $G$. If $\mathcal{D} \equiv \mathbb{R}^{d}$ and $D(\mathbf{x})$ is a second-order stationary field, i.e., if its mean $\left(\bar{D}=D_{0}\right)$ and variance $\left(\sigma_{D}^{2}\right)$ are constant, then $G=G\left(\mathbf{x}-\mathbf{x}^{\prime}, t-t^{\prime}\right)$ is given by the Gaussian function (9). An alternative to this approximation of the kernel $\kappa\left(\mathbf{x}, \mathbf{x}^{\prime}, t-t^{\prime}\right)$ is to treat it as a phenomenological transfer function in the spirit of the approaches described in the subsequent sections.

Nonlocal analogues of (60) for non-Fickian advection-dispersion transport can be found in Fiori et al. (2007), Koch and Brady (1988), Morales-Casique et al. (2006), Neuman (1993), among others. 


\subsection{Continuous Time Random Walks}

The continuous time random walk (CTRW) (Montroll and Weiss 1965; Scher and Lax 1973) relaxes the condition that a particle's motion is a Markovian process. Particle motion is characterized by a stochastic recursion relation for both the particle position $X_{n}$ and particle time $T_{n}$ after $n$ random walk steps as $X_{n+1}=X_{n}+\xi_{n}$ and $T_{n+1}=T_{n}+\tau_{n}$. The space-time random displacements $\left(\xi_{n}, \tau_{n}\right)$ at subsequent steps are independent and distributed according to a joint $\operatorname{PDF} \Psi(x, t)$. This recursive relation is non-Markovian in time but describes a Markov process in terms of its evolution in step number $n$. Thus, it is also called a semiMarkov process.

For an uncoupled CTRW, $\Psi(x, t)=\Lambda(x) \Psi(t)$, with a sharply peaked transition density $\Lambda(x)$, an expansion similar to that used to derive (8) leads to a time nonlocal equation for the PDF, $f(x, t)$, of finding a particle at the space-time point $(x, t)$ or, equivalently, for $c(x, t)$, the concentration of particles (Kenkre et al. 1973; Metzler and Klafter 2000),

$$
\frac{\partial f}{\partial t}=\int_{0}^{t} \kappa\left(t-t^{\prime}\right) \frac{\partial^{2} f}{\partial x^{2}}\left(x, t^{\prime}\right) \mathrm{dt}^{\prime} .
$$

The diffusion kernel $\kappa$ is defined in terms of the transition times PDF $\Psi(t)$ by

$$
\kappa(t)=\frac{1}{2}\left\langle\xi^{2}\right\rangle \mathcal{K}(t), \quad \hat{\mathcal{K}}(\lambda)=\frac{\lambda \hat{\Psi}(\lambda)}{1-\hat{\Psi}(\lambda)},
$$

where, for any appropriate function $g(t), \hat{g}(\lambda)$ indicates its Laplace transform; and $\left\langle\xi^{2}\right\rangle$ is the variance of $\xi_{n}$ for all $n \geq 1$. The transition time PDF $\Psi(t)$ has to be specified by the modeler from either prior knowledge or measurements of the state variable $f$. For a power-law distribution, $\Psi(t) \sim t^{-1-\beta}$ with $0<\beta<1$, the displacement variance scales as

$$
\left\langle X(t)^{2}\right\rangle \propto t^{\beta}, \quad 0<\beta<1,
$$

which indicates sub-linear scaling or sub-diffusive behavior.

CTRW is related to both Levy walk models (Metzler and Klafter 2000; Cushman et al. 2009) and fractional advection-dispersion equations (Benson et al. 2000; Cushman and Ginn 2000) that are characterized by spatiotemporal kernel functions with an asymptotic powerlaw scaling. Likewise, CTRW is connected to time-domain random walk models (Cvetkovic et al. 1996; Delay and Bodin 2001; Painter and Cvetkovic 2005) and multirate mass transfer models (Dentz and Berkowitz 2003).

\subsection{Matrix Diffusion and Multirate Mass Transfer}

The multirate mass transfer (MRMT) approach (Haggerty and Gorelick 1995; Carrera et al. 1998) separates the support scale into a mobile continuum and a suite of immobile continua, which communicate through a linear mass transfer. At each point, the immobile continua PDF $f_{\text {im }}(x, t)$ is related to its mobile continua PDF $f(x, t)$ through a linear relation (Carrera et al. 1998) $f_{\mathrm{im}}(x, t)=\int_{0}^{t} \phi\left(t-t^{\prime}\right) f\left(x, t^{\prime}\right) \mathrm{dt}^{\prime}$, where the kernel function $\phi(\cdot)$ is user-supplied. The evolution of the mobile continua PDF $f(x, t)$ is given by an integro-differential equation (Haggerty and Gorelick 1995) 
Fig. 6 Illustration of the memory functions for the slab-shaped (green) and spherical (blue) inclusions. The dash-dotted lines denote the preasymptotic $\sim t^{-1 / 2}$ behavior

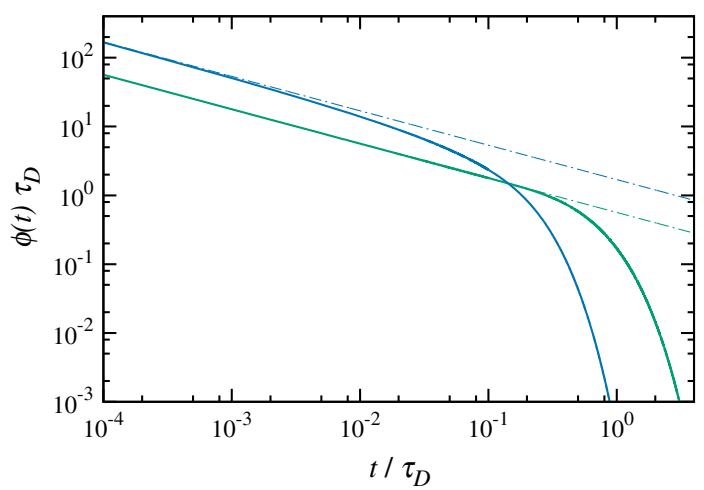

where $\epsilon$ is the ratio of the immobile and mobile volume fractions. The memory function $\phi(t)$ reflects the mass transfer mechanisms between the mobile and immobile continua.

For diffusive mass transfer into slab-shaped immobile regions, relevant for fracture matrix exchange, the memory function is given in terms of its Laplace transform as (Carrera et al. 1998)

$$
\hat{\phi}(\lambda)=\frac{\tanh \left(\sqrt{\lambda \tau_{D}}\right)}{\sqrt{\lambda \tau_{D}}},
$$

where $\tau_{D}$ is the characteristic diffusion time in the mobile domain. For diffusion in a medium characterized by spherical inclusions, it is given by

$$
\hat{\phi}(\lambda)=\frac{3}{\sqrt{\lambda \tau_{D}}}\left[\operatorname{coth}\left(\sqrt{\lambda \tau_{D}}\right)-\frac{1}{\sqrt{\lambda \tau_{D}}}\right] .
$$

These memory functions behave as $\phi(t) \propto t^{-1 / 2}$ for $t \ll \tau_{D}$ and decay exponentially fast for $t \gg \tau_{D}$. The displacement variance for times $t \ll \tau_{D}$ behaves sub-diffusively as (Bouchaud and Georges 1990)

$$
\left\langle X(t)^{2}\right\rangle \propto t^{1 / 2}
$$

and evolves diffusively for time $t \gg \tau_{D}$, as shown in Fig. 6. For diffusive mass transfer into heterogeneous immobile regions, the memory function can be determined by solving a heterogeneous diffusion problem (Gouze et al. 2008). For a heterogeneous medium composed of immobile regions with different diffusion properties, the memory function may behave as $\phi(t) \propto t^{\gamma}$ with $0<\gamma<1$ before a certain cutoff time.

Both the CTRW and MRMT frameworks have similar phenomenology as both account for memory effects due to a distribution of characteristic mass transfer timescales (Dentz and Berkowitz 2003; Dentz et al. 2012). In fact, it can be shown that the exponents in the power-law scalings of the memory function and transition time distribution are the same, $\beta=\gamma$. 


\section{Summary}

This paper provides a brief review of diffusion phenomena in porous media. It starts with the description of Brownian motion in a free fluid by the Langevin equation and its equivalence to the diffusion equation. We discuss the fundamental mechanisms of diffusion and derive the Einstein-Smoluchowski relations, which connect microscopic velocity fluctuations and energy dissipation.

We review the generalization of the diffusion concept from point-size inert particles to ensembles of finite number of particles of finite size, which leads to concentration-dependent diffusion coefficients and in this sense to non-Fickian diffusion. The Brownian motion of ions and in general charged particles is described by the Nernst-Planck equation.

The impact of diffusion on the rates of fast bimolecular chemical reactions can be quantified by the Smoluchowski theory, which relates the reaction rate directly to the diffusion rates of the reacting particles. For heterogeneous initial reactant concentration, the OvchinnikovZeldovich mechanism explains the slowing down of bimolecular chemical reactions due to segregation.

We then consider diffusion processes in porous media. Knudsen and Fick-Jacobs diffusion describe molecular diffusion under confinement. For diffusion in heterogeneous porous media, we distinguish between trap and barrier models, which have distinctly different transport properties. Trap models are related to diffusion problems under heterogeneous linear adsorption, while barrier models describe Fickian diffusion under spatial heterogeneity.

We furthermore discuss diffusion-like phenomena in porous media. These include Darcyscale flow models, which take the form of linear (single phase) and nonlinear (multiphase) diffusion equations, and dispersive transport of solutes. The concept of dispersion shares the same phenomenology as diffusion in that it models particle motion due to small-scale velocity fluctuations as a random walk and in this sense as a large-scale Brownian motion.

We conclude this brief review with an overview of phenomena and models for anomalous diffusion in porous media.

Acknowledgements This research was supported in part by US National Science Foundation (NSF) under Award Number CBET-1606192 issued to DMT, and by the European Research Council (ERC) through the Project MHetScale (617511) issued to MD.

\section{References}

Batchelor, G.K.: Brownian diffusion of particles with hydrodynamic interaction. J. Fluid Mech. 74(1), 1-29 (1976). https://doi.org/10.1017/S0022112076001663

Battiato, I., Ferrero V, P.T., O’ Malley, D., Miller, C.T., Takhar, P.S., Valdes-Parada, F.J., Wood, B.D.: Theory and applications of macroscale models. Transp. Porous Media (2019) (under review)

Bear, J.: Dynamics of Fluids in Porous Media. Elsevier, New York (1972)

Benson, D.A., Wheatcraft, S.W., Meerschaert, M.M.: The fractional-order governing equation of Levy motion. Water Resour. Res. 36(6), 1413-1423 (2000)

Bijeljic, B., Blunt, M.J.: Pore-scale modeling and continuous time random walk analysis of dispersion in porous media. Water Resour. Res. 42, W01,202 (2006)

Bouchaud, J.P., Georges, A.: Anomalous diffusion in disordered media: statistical mechanisms, models and physical applications. Phys. Rep. 195(4,5), 127-293 (1990)

Bruna, M., Chapman, S.J.: Excluded-volume effects in the diffusion of hard spheres. Phys. Rev. E 85, 011-103 (2012). https://doi.org/10.1103/PhysRevE.85.011103

Bruna, M., Chapman, S.J.: Diffusion of finite-size particles in confined geometries. Bull. Math. Biol. 76, 947-982 (2014). https://doi.org/10.1007/s11538-013-9847-0 
Burada, P.S., Hänggi, P., Marchesoni, F., Schmid, G., Talkner, P.: Diffusion in confined geometries. Chem. Phys. Chem. 10, 45-54 (2009). https://doi.org/10.1002/cphc.200800526

Carrera, J., Sanchez-Vila, X., Benet, I., Medina, A., Galarza, G., Guimera, J.: On matrix diffusion. Formulations, solutions methods and qualitative effects. Hydrogeol. J. 6(1), 178-190 (1998)

Cushman, J.H.: The Physics of Fluids in Hierarchical Porous Media: Angstroms to Miles. Kluwer Academic Publisher, New York (1997)

Cushman, J.H., Ginn, T.R.: Fractional advection-dispersion equation: a classical mass balance with convolution-Fickian flux. Water Resour. Res. 36(12), 3763-3766 (2000)

Cushman, J.H., Moroni, M.: Statistical mechanics with 3D-PTV experiments in the study of anomalous dispersion: part I. Theory. Phys. Fluids 13(1), 75-80 (2001)

Cushman, J.H., O’Malley, D.: Fickian dispersion is anomalous. J. Hydrol. 531, 161-167 (2015)

Cushman, J.H., O'Malley, D., Park, M.: Anomalous diffusion as modeled by a nonstationary extension of Brownian motion. Phys. Rev. E 79(3), 032-101 (2009). https://doi.org/10.1103/PhysRevE.79.032101

Cvetkovic, V., Cheng, H., Wen, X.H.: Analysis of nonlinear effects on tracer migration in heterogeneous aquifers using Lagrangian travel time statistics. Water Resour. Res. 32(6), 1671-1680 (1996)

Dagan, G.: Solute transport in heterogenous porous formations. J. Fluid Mech. 145, 151-177 (1984)

Dagan, G.: Flow and Transport in Porous Formations, 2nd edn. Springer, New York (2012)

Dagan, G., Neuman, S.P. (eds.): Subsurface Flow and Transport: A Stochastic Approach. Cambridge, New York (1997)

Delay, F., Bodin, J.: Time domain random walk method to simulate transport by advection-diffusion and matrix diffusion in fracture networks. Geophys. Res. Lett. 28, 4051-4054 (2001)

Dentz, M., Berkowitz, B.: Transport behavior of a passive solute in continuous time random walks and multirate mass transfer. Water Resour. Res. 39(5), 1111 (2003)

Dentz, M., Gouze, P., Russian, A., Dweik, J., Delay, F.: Diffusion and trapping in heterogeneous media: an inhomogeneous continuous time random walk approach. Adv. Water Resour. 49, 13-22 (2012)

Deshmukh, A., Elimelech, M.: Understanding the impact of membrane properties and transport phenomena on the energetic performance of membrane distillation desalination. J. Membr. Sci. 539, 458-474 (2017)

Dorfman, K.D., Yariv, E.: Assessing corrections to the Fick-Jacobs equation. J. Chem. Phys. 141, 044-118 (2014). https://doi.org/10.1063/1.4890740

Einstein, A.: Über die von der molekularkinetischen Theorie der Wärme geforderte Bewegung von in ruhenden Flüssigkeiten suspendierten Teilchen. Ann. Phys. 322, 549-560 (1905)

Fiori, A., Janković, I., Dagan, G., Cvetković, V.: Ergodic transport through aquifers of non? Gaussian log conductivity distribution and occurrence of anomalous behavior. Water Resour. Res. 43(9), W09,407 (2007). https://doi.org/10.1029/2007WR005976

Gardiner, C.: Stochastic Methods. Springer, Heidelberg (2010)

Gouze, P., Melean, Z., Le Borgne, T., Dentz, M., Carrera, J.: Non-Fickian dispersion in porous media explained by heterogeneous microscale matrix diffusion. Water Resour. Res. 44, W11,416 (2008)

Haggerty, R., Gorelick, S.M.: Multiple-rate mass transfer for modeling diffusion and surface reactions in media with pore-scale heterogeneity. Water Resour. Res. 31(10), 2383-2400 (1995)

Havlin, S., Ben-Avraham, D.: Diffusion in disordered media. Adv. Phys. 51, 187-292 (2002)

Jacobs, M.H.: Diffusion Processes. Springer, New York (1967)

Kane, J.J., Matthews, A.C., Orme, C.J., Contescu, C.I., Swank, W.D., Windes, W.E.: Effective gaseous diffusion coefficients of select ultra-fine, super-fine and medium grain nuclear graphite. Carbon 136, 369-379 (2018)

Kang, K., Redner, S.: Fluctuation-dominated kinetics in diffusion-controlled reactions. Phys. Rev. A 32, 435447 (1985)

Kayser, R.F., Hubbard, J.B.: Diffusion in a random medium with a random distribution of static traps. Phys. Rev. Lett. 51, 79-82 (1983)

Kenkre, V.M., Montroll, E.W., Shlesinger, M.F.: Generalized master equations for continuous-time random walks. J. Stat. Phys. 9(1), 45-50 (1973)

Kennard, E.H.: Kinetic Theory of Gases. McGraw-Hill, New York (1938)

Klinzing, G.R., Zavaliangos, A.: A simplified model of moisture transport in hydrophilic porous media with applications to pharmaceutical tablets. J. Pharamceutical Sci. 105(8), 2410-2418 (2016)

Koch, D.L., Brady, J.F.: Anomalous diffusion in heterogeneous porous media. Phys. Fluids 31(5), 965 (1988). https://doi.org/10.1063/1.866716

Kubo, R.: The fluctuation-dissipation theorem. Rep. Progr. Phys. 29(1), 255-284 (1966). https://doi.org/10. 1088/0034-4885/29/1/306

Langevin, P.: Sur la théorie du mouvement brownien. C. R. Acad. Sci. (Paris) 146, 530-533 (1908)

Metzler, R., Klafter, J.: The random walk's guide to anomalous diffusion: a fractional dynamics approach. Phys. Rep. 339(1), 1-77 (2000) 
Mitkov, I., Tartakovsky, D.M., Winter, C.L.: Dynamics of wetting fronts in porous media. Phys. Rev. E 58(5), 5245R-5248R (1998)

Montroll, E.W., Weiss, G.H.: Random walks on lattices. II. J. Math. Phys. 6(2), 167 (1965)

Morales-Casique, E., Neuman, S.P., Guadagnini, A.: Nonlocal and localized analyses of nonreactive solute transport in bounded randomly heterogeneous porous media: theoretical framework. Adv. Water Resour. 29(8), 1238-1255 (2006)

Moroni, M., Cushman, J.H.: Statistical mechanics with 3D-PTV experiments in the study of anomalous dispersion: part II. Experiment. Phys. Fluids 13(1), 81-91 (2001)

Narasimhan, T.N.: Fourier's heat conduction equation: history, influence, and connections. Rev. Geophys. 37(1), 151-172 (1999)

Neuman, S.P.: Eulerian-Lagrangian theory of transport in space-time nonstationary velocity fields: exact nonlocal formalism by conditional moments and weak approximation. Water Resour. Res. 29(3), 633645 (1993). https://doi.org/10.1029/92WR02306

Neuman, S.P., Tartakovsky, D.M.: Perspective on theories of anomalous transport in heterogeneous media. Adv. Water Resour. 32(5), 670-680 (2009). https://doi.org/10.1016/j.advwatres.2008.08.005

Neuman, S.P., Tartakovsky, D.M., Wallstrom, T.C., Winter, C.L.: Correction to the Neuman and Orr "Nonlocal theory of steady state flow in randomly heterogeneous media". Water Resour. Res. 32(5), 1479-1480 (1996)

O’Malley, D., Cushman, J.H.: A renormalization group classification of nonstationary and/or infinite second moment diffusive processes. J. Stat. Phys. 146, 989-1000 (2012)

O’Malley, D., Vesselinov, V.V., Cushman, J.H.: A method for identifying diffusive trajectories with stochastic models. J. Stat. Phys. 156(5), 896-907 (2014)

Ovchinnikov, A.A., Zeldovich, Y.B.: Role of density fluctuations in bimolecular reaction kinetics. Chem. Phys. 28, 215-218 (1978)

Painter, S., Cvetkovic, V.: Upscaling discrete fracture network simulations: an alternative to continuum transport models. Water Resour. Res. 41(2) (2005). https://doi.org/10.1029/2004WR003682

Pfannkuch, H.O.: Contribution a l'étude des déplacements de fluides miscibles dans un milieux poreux. Rev. Inst. Fr. Petr. 18, 215-270 (1963)

Picchi, D., Battiato, I.: The impact of pore-scale flow regimes on upscaling of immiscible two-phase flow in porous media. Water Resour. Res. 54(9), 6683-6707 (2018)

Picchi, D., Ullmann, A., Brauner, N.: Modelling of core-annular and plug flows of Newtonian/non-Newtonian shear-thinning fluids in pipes and capillary tubes. Int. J. Multiph. Flow 103, 43-60 (2018)

Qian, G., Morgan, N.T., Holmes, R.J., Cussler, E.L., Blaylock, D.W., Froese, R.D.J.: Sublimation as a function of diffusion. AIChE J. 62(3), 861-867 (2016)

Redner, S.: A Guide to First-Passage Processes. Cambridge University Press, Cambridge (2001)

Regner, B.M., Tartakovsky, D.M., Sejnowski, T.J.: Identifying transport behavior of single-molecule trajectories. Biophys. J. 107, 2345-2351 (2014). https://doi.org/10.1016/j.bpj.2014.10.005

Regner, B.M., Vucinic, D., Domnisoru, C., Bartol, T.M., Hetzer, M.W., Tartakovsky, D.M., Sejnowski, T.J.: Anomalous diffusion of single particles in cytoplasm. Biophys. J. 104, 1652-1660 (2013)

Risken, H.: The Fokker-Planck Equation. Springer, Heidelberg (1996)

Scher, H., Lax, M.: Stochastic transport in a disordered solid. I. Theory. Phys. Rev. B 7(1), 4491-4502 (1973)

Sutherland, W.: A dynamical theory of diffusion for non-electrolytes and the molecular mass of albumin. Phil. Mag. S. 9, 781-785 (1905)

Toussaint, D., Wilczek, F.: Particle-antiparticle annihilation in diffusive motion. J. Chem. Phys. 78, 2642-2647 (1983)

van der Pas, P.W.: The discovery of the Brownian motion. Scientiarum Historia 13, 27-35 (1971)

von Smoluchowski, M.: Zur kinetischen Theorie der Brownschen Molekularbewegung und der Suspensionen. Ann. Phys. 21, 757-780 (1906)

von Smoluchowski, M.: Versuch einer mathematischen Theorie der Koagulationskinetik kolloidaler Lösungen. Z. Phys. Chem. 92, 129-168 (1917)

Zalc, J.M., Reyes, S.C., Iglesia, E.: The effects of diffusion mechanism and void structure on transport rates and tortuosity factors in complex porous structures. Chem. Eng. Sci. 59, 2947-2960 (2004). https://doi. org/10.1016/j.ces.2004.04.028

Zhang, X., Tartakovsky, D.M.: Effective ion diffusion in charged nanoporous materials. J. Electrochem. Soc. 164(4), E53-E61 (2017)

Zhang, X., Urita, K., Moriguchi, I., Tartakovsky, D.M.: Design of nanoporous materials with optimal sorption capacity. J. Appl. Phys. 117(24), 244-304 (2015). https://doi.org/10.1063/1.4923057

Publisher's Note Springer Nature remains neutral with regard to jurisdictional claims in published maps and institutional affiliations. 